\title{
On The Possibility of Improving the Reliability of Communication in Fiber-Optic Systems with Spectral Compaction
}

\author{
Masharipov Otoboy Matyoqubovich
}

\begin{abstract}
In the article, the authors present a concrete example of a block diagram of a signal transmission device at WDM. Operation of this device with the representation of plots of pulse diagram of operation at standard characteristics of services and evaluation of their quality indicators depending on the fault tolerance in the whole system under study. Recommendations for practical use in various types of network topology are given. Spectral compaction technologies such as CWDM, DWDM, HDWDM, principles of construction of these technologies, as well as advantages and disadvantages are considered. The functional schemes of the signal transmission device and the functional scheme of the optical transceiver and the principle of operation of these devices are given. The frequency plan for CWDM systems is defined by ITU G. 694.2. The scope of CWDM technology is urban networks with a distance of up to $50 \mathrm{~km}$. The advantage of this type of WDM systems is low (compared to other types) the cost of equipment due to lower requirements for the constituent equipment of the fiber optic network. The frequency plan for DWDM systems is defined by ITU G. 694.1. The field of application - backbone network. This kind of WDM systems has higher requirements for components than CWDM (radiation source spectrum width, source temperature stabilization, etc.). The impetus for the rapid development of DWDM networks was given by the appearance of inexpensive and efficient fiber erbium amplifiers (EDFA) operating in the range from 1525 to $1565 \mathrm{~nm}$ (the third window of transparency of quartz fiber). For simplicity, the model is limited to a frequency plan DWDM with a step of 100 $\mathrm{GHz}$, and CWDM has eight main channels in the range of 1470-1610 $\mathrm{nm}$. The calculations of reliability indicators of the basic version and the modified version of the signal transmission device are given.
\end{abstract}

Keywords : wdm, cwdm, dwdm, hdwdm, trigger, decoder, logic elements.

\section{INTRODUCTION}

Currently, the most promising is the spectral method of channel sealing, carried out in accordance with the international standard [1]. This method allows you to simultaneously transmit several information channels on one optical fiber at different carrier frequencies. WDM (eng. Wavelength-division multiplexing (WDM, literally

Revised Manuscript Received on February 05, 2020.

* Correspondence Author

Masharipov Otoboy Matyoqubovich*, Doctoral Department of Telecommunications Engineering, Urgench branch of Tashkent University of Information Technologies named After Muhammad al-Khwarizmi.

Email: Otaboy_1963@mail.ru

(C) The Authors. Published by Blue Eyes Intelligence Engineering and Sciences Publication (BEIESP). This is an open access article under the CC BY-NC-ND license (http://creativecommons.org/licenses/by-nc-nd/4.0/) wavelength division multiplexing) is a technology that allows multiple information channels to be transmitted simultaneously over a single optical fiber at different carrier frequencies. This allows you to significantly increase the bandwidth of the channel. Moreover, it allows the use of already laid fiber-optic lines [7]. Thanks to WDM it is possible to organize two-way multichannel traffic transmission on one fiber. In the simplest case, each laser transmitter generates a signal at a specific frequency from the frequency plan. All these signals are combined by a multiplexer (MUX) before being introduced into the optical fiber. At the receiving end, the signals are similarly separated by a demultiplexer (DEMUX). Here, as in SDH networks, the multiplexer is the key element. Historically, the first two wave WDM systems operating at the Central wavelengths-from the second and third Windows of fiber transparency (1310 and $1550 \mathrm{~nm}$ ). The main advantage of such systems is that due to the large spectral spacing there is no influence of channels on each other. This method allows you to either double the transmission speed on a single optical fiber, or to organize a duplex connection. WDM technology based on ITU-T Rec standard frequency plan. On the street.692 can be divided into three groups. Coarse WDM (Coarse WDM - CWDM) - systems with a frequency difference of at least $200 \mathrm{GHz}$, allowing multiplexing of no more than 18 channels. Currently, CWDM operate in the band from $1270 \mathrm{~nm}$ to $1610 \mathrm{~nm}$, the gap between the channels is 20nm (200Ghz), 16 spectral channels can be multiplexed. Dense WDM (dense WDM — DWDM) - systems with channel spacing of at least $100 \mathrm{GHz}$, allowing multiplexing no more than 40 channels. High density WDM (high density WDM - HDWDM) - systems with channel spacing of 50 $\mathrm{GHz}$ or less, allowing multiplexing of at least 64 channels. The frequency plan for CWDM systems is defined by ITU G. 694.2. Application of CWDM technology-urban networks with a distance of up to $50 \mathrm{~km}$. the Advantage of this type of WDM systems is low (compared to other types) the cost of equipment due to lower requirements for the constituent equipment of the fiber optic network. The frequency plan for DWDM systems is defined by ITU G. 694.1. The field of application - backbone network. This kind of WDM systems has higher requirements for components than CWDM (radiation source spectrum width, source temperature stabilization, etc.). The impetus for the rapid development of DWDM networks was given by the appearance of inexpensive and efficient fiber erbium amplifiers (EDFA) operating in the range from 1525 to 1565 $\mathrm{nm}$ (the third window of transparency of quartz fiber). 


\section{On The Possibility of Improving the Reliability of Communication in Fiber-Optic Systems with Spectral Compaction}

For simplicity of the model we will limit ourselves to the frequency plan DWDM with a step of $100 \mathrm{GHz}$, and in CWDM we will consider eight main channels in the range of $1470-1610 \mathrm{~nm}$. For figure. 1 both plans are given with the indication of the area of intersection, where on the ordinate axis the attenuation is postponed, and on the abscissa axis-the wavelength of radiation. In the upper part of the figure shows the DWDM plan with a step of $0.8 \mathrm{~nm}$ (band $\mathrm{C}$ ) and the intersecting area, in the lower-CWDM plan with a step of 20 $\mathrm{nm}$, and on it the positions of bands $\mathrm{C}$ and $\mathrm{l}$ of the DWDM plan. It is known that each of the bands $\mathrm{C}$ and $\mathrm{L}$ can be placed on 41 standard channels with a step of $100 \mathrm{GHz}[5,6]$.

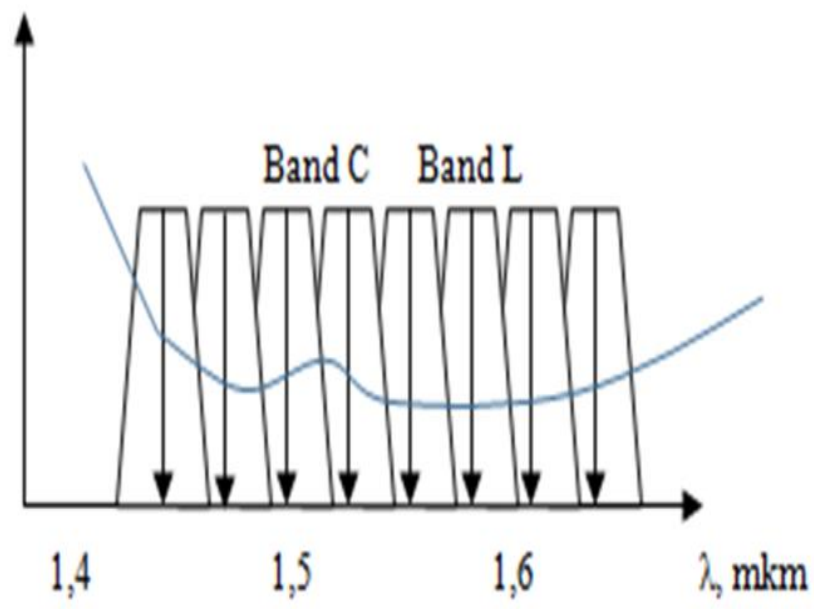

Figure.1 CWDM and DWDM Wave plans

Along with this, there is a problem of improving the reliability of communication in all areas of the fiber-optic communication system (FOCS) with WDM, using new approaches, ways, means to ensure the required guaranteed uptime. Therefore, in the context of the latter, the solution of this problem becomes relevant and timely.

\section{PROBLEM STATEMENT}

Using a signal transmission device to look for ways to improve the reliability of digital fiber optic transmission systems.

The authors of this article presents a modified version of the principle of operation of one of the hardware solutions in the form of a signal transmission device (STD) used to improve the reliability of communication in FOCS at WDM.

The purpose of this device [2], in FOCS with WDM to provide the required indicators of fault tolerance while maintaining high performance and quality of information. It is achieved by determining the direction in which the requested station is located, sending an optical signal only in this direction and blocking the transmission and reception of messages for other stations. The proposed device contains in its composition of the aggregate number of elements, both electronic and optical, and combinations thereof. For figure. 2 and 3 , the functional diagram of the device and the block diagram of the optical reception-transmitter are given.

For figure.2 the functional diagram of STD is shown.

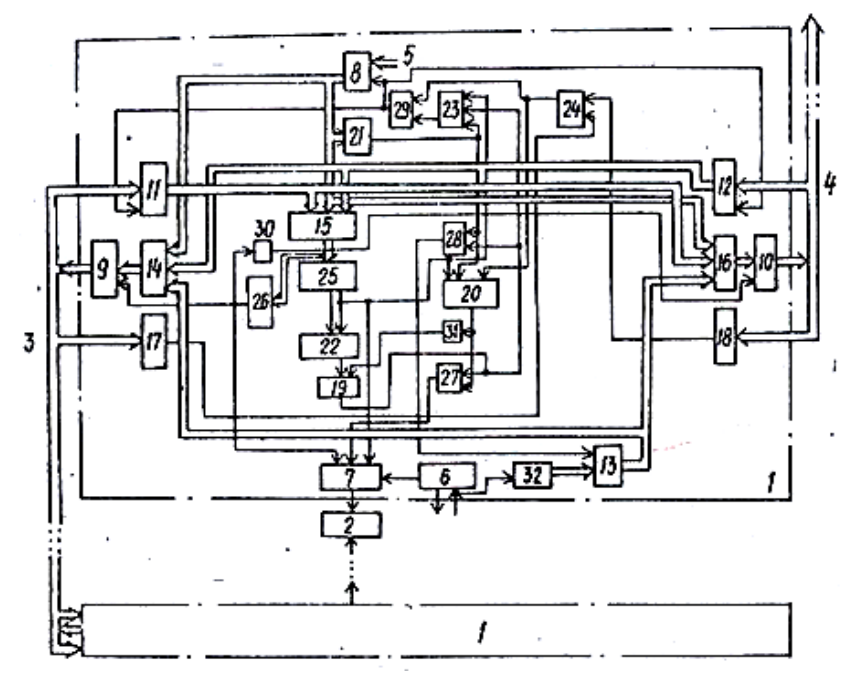

Figure.2 STD Function diagram.

The functional diagram of the optical transceiver is shown in Fig.3. The signal transmission device comprises: $\mathrm{N}$ stations 1, fiber light guides 2, control buses 3 and 4, ADRs buses 5 , an optical transceiver 6 , an optical switch 7 , element blocks and 8-13, element blocks OR 14-15, elements and 17-19, elements or 20-24, decoders 25 and 26, triggers 27-29, inverters 30-31 and a signal generation unit for information exchange. The optical transceiver 6 comprises an emitter 33, a modulator 34, a photodetector 35, and an amplifier 36. The signal transmission device works as follows. For information exchange between $\mathrm{i}$-th and $\mathrm{j}$-th stations 1 through block 8 elements And the electrical signals that determine the number $\mathrm{j}$ of the requested station 1 simultaneously arrive at the inputs of the OR element 21, to the inputs 14, 15 and 16 of the blocks OR elements. At the output of the element or 21, a signal "Log.1" simultaneously coming through the element or 23 to the input of the trigger 29 and throwing it into the state" Log.0", to the second input of trigger 28 and through element OR 20 to the first input of trigger 27. The signal from the output of the trigger 29 is simultaneously fed to the control inputs 8, 11 and 12 of the element blocks And, thereby prohibiting the address input from the address input bus 5 and the reception of signals from the control buses 3 and 4 until the end of the information exchange, respectively.

Signal level " Log.1", coming from the output of the trigger 28 to the control input of block elements And 13, permits the operation of unit 32-signal end information exchange, and a signal of level "Log.0 " coming from the trigger output 27, sets the optical switch 7 to the "Transmit" position.

Electrical signals coming from the bus 5 input address of the requested station 1 , pass through 14 and 16 blocks of elements OR, inputs 9 and 10 blocks of elements and respectively, as well as block elements or 15 come to the input of the decoder 26, which, depending on the address of the requested station 1 determines the direction of its location. The output of the decoder 26 is formed a control signal supplied to the second control input of the optical switch 7, the control input of block elements And 9, and also via an inverter 30, to the control input of the block elements And 10, 
And opens the unit, whose direction coincides with the direction in which is located the requested station 1 . At receipt of the electric signals defining number of $\mathrm{j}$-th station 1 , on buses 3 and 4 of management on inputs 11 or 12 blocks of elements And 2-th station 1 they through the block of elements or 15 pass on an input of the decoder 26 defining the further direction of search, and on information inputs 9 and 10 blocks of elements And.1 "comes to the input of the element And 19, the second input of which from the output of the inverter 31 is also fed a signal" Log.1". Based on what is said at the output of the element And 19 there is a level signal " Log.1", which simultaneously enters the first input of trigger 28, the second input of element OR 23 and the second input of trigger 27. The trigger 28 is thrown into a state of "Log. 0 " and thereby disconnects the signal generation unit of the end of the information exchange from the bus input signal of the end of the information exchange. Signal "Log.1" from the second input of the element OR 23 passes to the first input of the trigger 29, switching it to a zero state, prohibiting input through the block of elements And 8 until the end of the information exchange. At the output of trigger 27, the signal "Log.1", which sets the optical switch 7 to the "Relay" position. In case of receipt of the electric signals defining number of $j$-th station 1 , on buses 3 and 4 of management on inputs 11 or 12 blocks of elements And j-th station 1, they pass through the block of elements OR 15 on an input of the decoder 25 . At the output of the decoder 25 , according to the recognition of its own address, a signal " Log.1", coming simultaneously to the third control input of the optical switch 7. Next, through the element OR 20 to the first input of the trigger 27, throwing it into the state " Log.0" and thereby an optical switch 7 in position "Welcome" and also through the third input element OR 23 to a first input of the trigger 29 forming the control signal by the unit elements And 8 prohibiting the input address until the end of the information exchangeThe emitter of the optical transceiver 6 of the I-th station 1 generates light signals coming to the first input of the modulator 34 , the second input of which receives electrical information signals. The modulated optical signals from the output of the modulator 34 are fed through the j-th optical switch 7 to the fiber light guide 2. Since only the optical switch 7 of the I-th station 1 is open for receiving light signals, the light signals from the fiber light guide 2 arrive at the input-output of the transceiver 6 only of the I-th station 1 and then at the input of the photodetector 35. From the output of the photodetector 35 , the restored sequence of electrical signals through the amplifier 36 enters the output of the transceiver 6 . The signal about the end of the information exchange is formed as follows.

When sending a certain amount of "Log.0" block 32formirovaniya signal the end of the information exchange generates a code sent on the bus input signal the end of the information exchange, through the block elements or 14, block elements and 9 or block elements OR 16 and block elements and 10 in the bus 3 and 4 control. When receiving a signal about the end of the information exchange at the output 17 or 18 element and formed a signal "Log. 1 " coming through the element OR 20 to the first input of the trigger 27 setting the optical switch 7 to the "Receive-transmit" position"

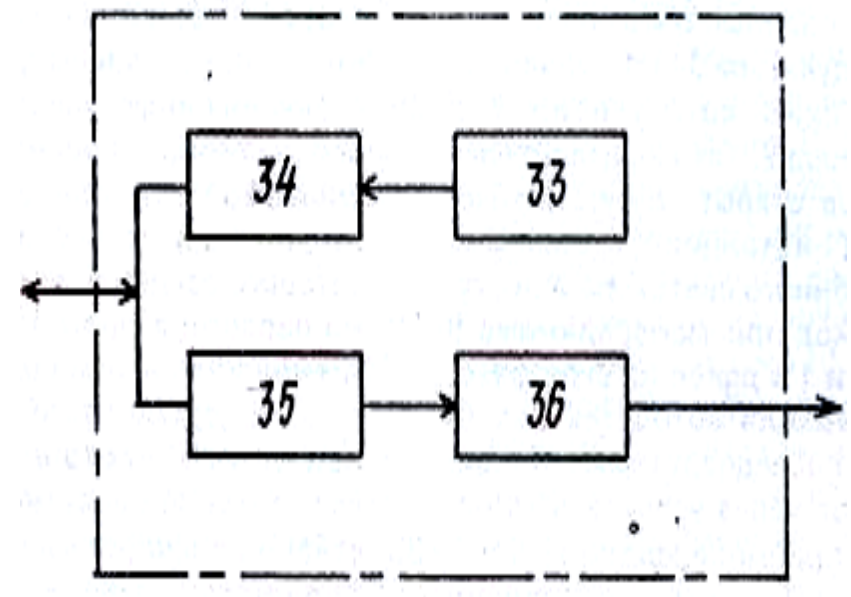

Figure.3 Functional diagram of the optical transceiver.

The optical transceiver 6 comprises an emitter 33 'a modulator 34' a photodetector 35 and an amplifier 36.

Therefore, it will be necessary to assess the degree of its impact on the reliability of FOCS with WDM with the proposed device. To do this, it is required to calculate and estimate the failure rate, summing all homogeneous elements (ICS) and transmitting and receiving means, according to the following formulas [3]:

$$
\begin{gathered}
\lambda_{1}(\mathrm{t})=\sum_{i=1}^{n} \lambda(t)(1) \quad \lambda 2(t)=\sum_{i=1}^{n} \lambda \operatorname{rec}(t) \\
\lambda_{3}(\mathrm{t})=\sum_{i=1}^{n} \lambda \operatorname{tran}(t)
\end{gathered}
$$

where: $\lambda$ 1(t) - failure rate block elements matrix products", sum matrix» $\lambda_{2(\mathrm{t}), \text { и }} \lambda_{3(\mathrm{t})}$ - failure rates of receiver and transmitter respectively.

The main characteristic of the reliability of the UPS is the probability $\mathrm{P}(\mathrm{t})$ of its uptime during time $\mathrm{t}$. the Sum of the

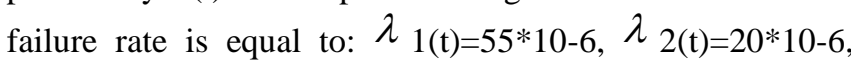
$\lambda_{3(\mathrm{t})}=20 * 10-6$. Given the amount of intensity $\lambda(t)$ failures, i.e. the number of failures per unit of time we determine the probability of failure-free operation within 2000 hours: $\mathrm{P}(2000)=\mathrm{e}-\lambda(t)$

$$
\mathrm{P}(\mathrm{t})=\mathrm{e}-\lambda(t) * *_{\mathrm{t}}=\mathrm{e}-55 * 10-6 * 2000=0,8958
$$

\section{RESULT}

The results of FPGA fault tolerance calculation are presented in [8]. Taking advantage of them and the above results of calculation of the main indicators and characteristics, determining the average intensity and probability of failure-free operation of the basic version, we obtain the basic and modified versions, respectively. Which constitute respectively:

$$
\begin{gathered}
\lambda_{\mathrm{b}}(\mathrm{t})=55^{*} 10^{-6} 1 / \mathrm{h} \\
\mathrm{P}_{\mathrm{b}}(\mathrm{t})=\mathrm{e}^{-\lambda(t) * \mathrm{t}}=\mathrm{e}^{-55^{*} 10-6 * 2000}=0,8958 \\
\lambda_{\mathrm{p}}(\mathrm{t})=44,8 * 10^{-6} \quad 1 / \mathrm{h}
\end{gathered}
$$

Published By: 


$$
\mathrm{P}_{\mathrm{p}}(\mathrm{t})=\mathrm{e}^{-\lambda(t) *_{\mathrm{t}}}=\mathrm{e}^{-44,8 * 10-6 * 2000}=0,9231
$$

Comparison of these data allows us to judge that at the same time intervals (at $\mathrm{t}=2000 \mathrm{~h}$ ) the probability of failure-free operation of the modified version is higher than the base version. Therefore, the modification of the basic version of the STD significantly improve reliability when using FPGAs.

For figure. 4 shows the operation of this device with a representation of the plots of the pulse diagram of the work.

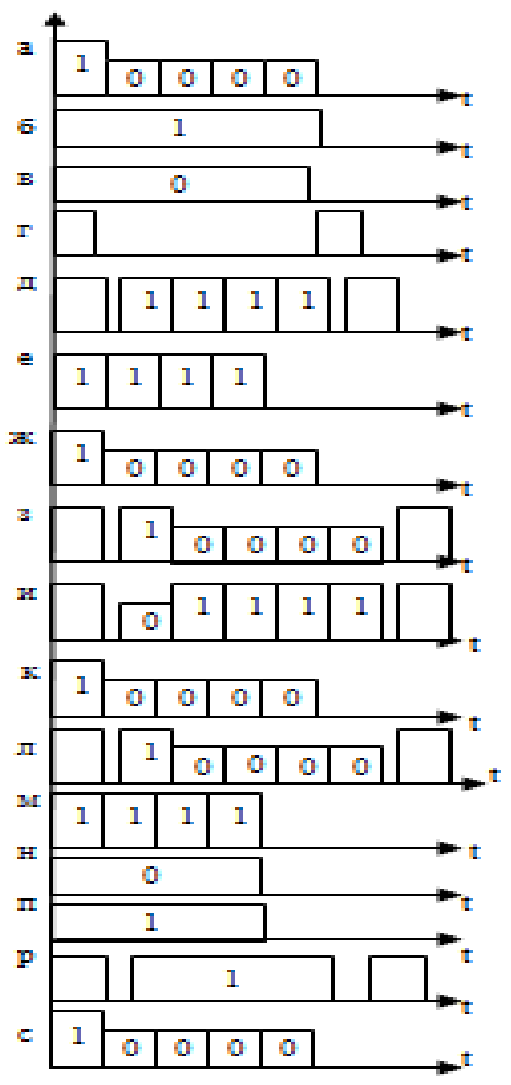

Figure. 4. Time - pulse diagram of the operation of STD Below are the failure rates of individual STD elements.

$$
\begin{aligned}
& \lambda(t)=0,6 * 10-6 * 6=3,6 * 10-61 \text { hour; } \\
& \lambda(\mathrm{t})=0,6 * 10-6 * 3=1,8 * 10-61 \text { hour; } \\
& \lambda(t)=0,6 * 10-6 * 3=1,8 * 10-61 \text { hour; } \\
& \lambda(t)=0,6 * 10-6 * 5=3 * 10-6 \text { 1hour; } \\
& \lambda(\mathrm{t})=0,6 * 10-6 * 2=1,2 * 10-6 \text { 1hour; } \\
& \lambda(\mathrm{t})=0,6 * 10-6 * 3=1,8 * 10-6 \text { 1hour; } \\
& \lambda(t)=0,6 * 10-6 * 2=1,2 * 10-6 \text { 1hour; } \\
& \lambda(\mathrm{t})=0,6 * 10-6 * 1=0,6 * 10-6 \text { 1hour; } \\
& \lambda(\mathrm{t})=20 * 10-6 * 2=40 * 10-6 \text { 1hour. }
\end{aligned}
$$

\section{CONCLUSION}

In conclusion, we can draw the following conclusions:

1. The introduction of new technologies and their principles, methods and tools that increase the efficiency of FOCL requires the development of adequate new measures and approaches to ensure its proper reliability.

2. The authors propose possible applications of the new upgraded version of the device, implemented on the basis of FPGAs.

3. Thus, the proposed device can find its application in zonal, local and corporate networks, as well as in urban fiber optic networks with the delivery of large amounts of data to the last "mile" with high reliability and efficiency of the communication channel.

4. Comparison of fault tolerance of the basic and modified versions of the UPS allows, judges that in the latter case, while maintaining the required fault tolerance, it is possible to increase the number of requested stations and the number of services provided. This makes it possible to increase the traffic of messages and services delivered to the last mile, with high quality indicators providing the required reliability of communication in the fiber in the spectral densification.

The modified device has the following form.

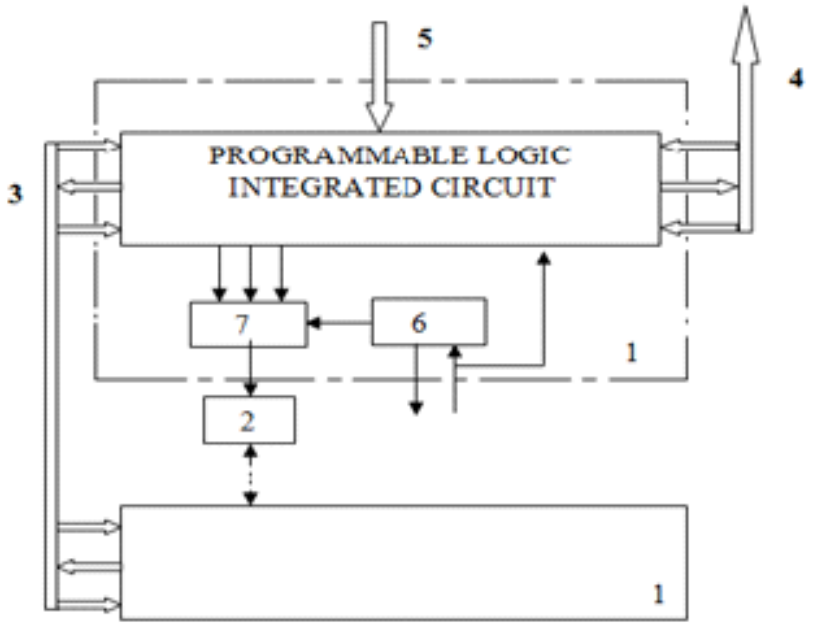

\section{Figure.5 Functional scheme of STD on the basis of FPGA}

As a result of upgrading the signal transmission device with FPGA, the reliability of the device increases. Calculation of reliability parameters is given above. This development is designed to improve the reliability of local fiber-optic communication systems.

\section{REFERENCES}

1. Slepov N. N. Modern technologies of digital fiber-optic communication networks.2001.

2. E. B. Makhmudov, D. V. Protopopov and E. N. Biktimirov A. Since 1688427. Signal transmission device. 1991.

3. Raikin A. L. Elements of the theory of reliability of technical systems 1978

4. E. B. Mahmudov. About one method of increase of reliability and reliability of communication. In the collection of works of FRRT TEIS edited by academician T. D. Rajabov RIS Publishing house, Tashkent 1993, art. pp 88-89

5. Masharipov O.M., Matyokubov U.K. Povыshenie nadejnosti dostavki informatsii pri spektralınom uplotnenii // Vestnik TUIT nauchno-texnicheskiy i informatsionno-analiticheskiy jurnal. №1, 2017. - p 67. (in Russian)

6. T.O. Rakhimov. Matlable modeling a camergion servicing system on Matlab program transmission. Journal of the Scientific and Practical and Information-Analytical Journal of Muhammad al-Khwarizmi № 1 (7), June 2019 at pp 42-45.

7. Matveevskiy V.R. Nadejnostb texnicheskix sistem. Uchebnoe posobie - Moskovskiy gosudarstvennыy institut elektroniki i matematiki. M., 2002 g. - p 113. (in Russian)

8. A.V. Listvin, V. N. Listvin, D. V. Shvyrkov Optical fibers for communication lines. - M.: Laserart, 2003

9. Network electronic scientific journal "system engineering" №7 2009.

10. Rakhimov Temurbek Omonboyevich, Ismailov Shavkat Kuzievich, Matyokubov Utkir Karimovich, Eschanov Umar Kutlimuratovich, Kuchkarov Voxid Alisherovich Modeling Discrete Channels Based on Gilbert Model using Matlab Software International Journal of Engineering and Advanced Technology (IJEAT) ISSN: 2249 - 8958, Volume-9 Issue-2, December, 2019. pp 3568-3571.

Published By: 
11. Masharipov O. M., Makhmudov E. B. Creating Checking Elements of Hamming Codes on a New Elemental Base Based on Programmable Logical Integral Schemes AKTA of Turin polytechnic university in Tashkent edition 1/2019. pp 60-63.

\section{AUTHORS PROFILE}

Masharipov Otaboy Matyoqubovich doctoral student of Telecommunications Engineering, Urgench branch of Tashkent University of Information Technologies named after Muhammad al-Khwarizmi.

Email: Otaboy 1963@mail.ru

Тел number: +998942393163 